\title{
Brain imaging in catatonia: systematic review and directions for future research
}

Alexandre Haroche ${ }^{1}$, Jonathan Rogers ${ }^{2,3}$, Marion Plaze ${ }^{1}$, Raphaël Gaillard ${ }^{1}$, Steve CR Williams ${ }^{4}$, Pierre Thomas 5 , Ali Amad ${ }^{4,5}$

1. Hôpital Sainte-Anne, Groupe Hospitalo-Universitaire, 75014, Paris, France.

2. Division of Psychiatry, University College London, London, UK

3. South London and Maudsley NHS Foundation Trust, London, UK

4. Department of Neuroimaging, Institute of Psychiatry, Psychology and Neuroscience, King's College London, London, UK.

5. Univ. Lille, CNRS, CHU LILLE, UMR9193-PsychiC-SCALab, Pôle de Psychiatrie, F-59000 Lille, France.

* Corresponding author: Alexandre Haroche Department of Psychiatry

Service Hospitalo-Universitaire, Centre Hospitalier Sainte-Anne, Paris, France

푤: 0152968000 ; mail : a.haroche@ghu-paris.fr

4910 words 


\section{INTRODUCTION}

Catatonia is an identifiable syndrome of psychomotor dysregulation first described by Karl Ludwig Kahlbaum in 1874 (Kahlbaum, 1874). Historically, catatonia was mainly associated with schizophrenia, and considered as a subtype of this disorder for most of the twentieth century. Since the 1970's, catatonia has been described as a complex syndrome involving motor, affective, behavioural and vegetative symptoms, which can be associated more broadly with affective disorders, schizophrenia and many other neuropsychiatric conditions (Fink, 2013). Its clinical manifestations are heterogeneous and no fewer than 40 signs have been described in one of the diagnostic scales (G. Northoff et al., 1999b). According to the fifth edition of Diagnostic and Statistical Manual of Mental Disorders (DSM) (American Psychiatric Association et al., 2013) the diagnosis of catatonic syndrome can be made when three or more symptoms from the following twelve are present : catalepsy, waxy flexibility, stupor, agitation, mutism, negativism, posturing, mannerisms, stereotypies, grimacing, echolalia, echopraxia (see box 1). The first-line treatment of catatonic syndrome is benzodiazepines, particularly lorazepam, which is effective in almost $80 \%$ of cases. Electroconvulsive therapy (ECT) is initiated in patients with catatonia who do not respond to benzodiazepines or when a decisive and rapid improvement is required (Fink and Taylor, 2006).

The prevalence of catatonia is generally considered to be between 10 and $25 \%$ of patients hospitalised in psychiatric wards (Walther and Strik, 2016). However, figures depend on the choice of the diagnostic criteria. For example, in a 2016 study of 87 hospitalized patients, the prevalence of catatonic syndrome varied from $3.4 \%$ with the Catatonia Rating Scale to $10.3 \%$ when the Bush Francis Catatonia Rating Scale was used (Sarkar et al., 2016). In a recent meta-analysis of 74 studies providing data collected from 1935 to 2017 across all continents, mean catatonia prevalence was $9.0 \%$ in clinical samples (Solmi et al., 2018).

The pathophysiology of catatonia is poorly understood. Interestingly, one of the first hypotheses implicated subcortical pathways, and more precisely a blockade at the level of the basal ganglia (Baruk and de Jong, 1930), because of the clinical similarity between catatonic and extrapyramidal syndromes. According to Carroll, catatonia is associated with excessive inhibition of the thalamus due to excessive inhibition of the external Globus Pallidum and insufficient inhibition of internal Globus Pallidum (iGP). This hypothesis can explain the harmful effect of antidopaminergic 
drugs (blockade at a striatal level) and the beneficial effect of GABAergic drugs (inhibition of iGP) (Carroll, 2000). Neuropathological findings have also highlighted the role of the basal ganglia (Northoff, 2000). Nevertheless, these studies yield inconsistent results and have generally been limited to patients with catatonia in the context of schizophrenia. Extrapolation to all catatonic syndromes is therefore questionable. Furthermore, clinical observations after lobotomies and frontal lobe lesions, and experiments performed on monkeys have highlighted the specificity of supplementary motor area (SMA) dysfunction in the occurrence of waxy flexibility and negativism (Joseph, 1999)

The most recent data suggest that the catatonic syndrome is a frequent, underestimated, and potentially lethal condition. However, the precise brain mechanisms that could be the source of the symptoms are very poorly known. Today, one can argue that the accessibility of brain imaging, and specifically functional neuroimaging, could be the starting point of a new era in the understanding of this condition. The objective of the present study is to provide an extensive review of all brain imaging studies in catatonia, and then, to present the main pathophysiological models presented currently considered in the literature. Finally, we discuss a potential framework for future brain imaging studies in catatonia. 


\section{METHODS}

To identify studies eligible for this review, a systematic search was conducted using the PubMed and Embase databases up to $10^{\text {th }}$ of August 2019, using a combination of search terms "catatonia" and either "brain imaging" or "neuroimaging". We also examined the reference sections from the selected papers to identify any additional relevant studies and asked other researchers in the field. Papers were included in the systematic review if (a) they were published in an English-language peer-reviewed journal; (b) the study enrolled patients with catatonic syndrome; (c) the diagnosis was made according to specified criteria, and (d) the studies included brain imaging. Article titles and the abstracts of studies identified from the searches were screened and excluded from the systematic review for the following reasons: inaccessible studies (even after contacting the authors); study not written in English; study unrelated to catatonic syndrome; review article, opinion, or hypothesis article; study without brain imaging; posters; incomplete data. The full text of studies that passed the initial screening was reviewed and potentially excluded based on the same criteria. We clustered the retained papers into case reports and group studies. The literature search strategy is summarized in the flow chart presented in Figure 1. 


\section{RESULTS}

A total of 505 studies were extracted from Embase, and 131 from PubMed. Sixty-four duplicates were automatically removed. A hundred and three studies were irrelevant based on titles and abstracts, 320 articles met the exclusion criteria, leaving a total of 149 studies, with 134 case reports (161 separate individuals reported) and 15 brain imaging group studies. Subsequent searches identified 5 supplementary group studies and 3 case reports.

\section{Case reports}

137 case reports were found including 161 patients who were mostly women (57\%), with a mean age of 39 years (range: 8 to 90 years). 139 patients had a cerebral structural Magnetic Resonance Imaging (MRI), 43 patients had a computerized tomography (CT) head, 18 patients had a Single Photon Emission Computerized Tomography (SPECT) and 18 had a Positron Emission Tomography (PET) scan. 22 patients had both an MRI and CT; 9 patients had both an MRI and PET; 7 patients had both an MRI and SPECT; 5 patients had an MRI; CT and SPECT; 4 patients had an MRI, CT, and PET; 3 patients had only a PET; 1 patient had a CT, MRI and SPECT; 1 patient had an MRI, SPECT and PET; 1 patient had a SPECT and PET; and 1 patient only had a SPECT. Most of the patients had an underlying condition that was not considered as only psychiatric (126 of 162 patients). In 45 patients, the main diagnosis was an inflammatory condition (10 lupus, 23 anti-NMDA receptor encephalitis, 8 encephalitis of other origin, 2 multiple sclerosis, 1 acute disseminated encephalomyelitis, 1 subacute sclerosing panencephalitis). An abnormality was found in at least one modality for 123 patients (76.4\%). The results of CT brain were abnormal in $44 \%$ of cases (19 of 43 ), and results were directly related to the final diagnosis in 26 $\%$ of these case reports (11 of 43 cases), meaning that conclusions of brain CT were not considered as incidental according to the diagnosis highlighted in the case report by the authors. Brain MRI scans were abnormal in $65 \%$ of cases (90 of 139), and directly related to the diagnosis in $47 \%$ of cases (66 of 139). Most of the cases reported diffuse or multiple abnormalities, such as miliary brain metastasis (Dziadziuszko et al., 2014), or nonspecific white matter abnormalities (Vancaester and Santens, 2007). On the 18 PET scans, 17 were abnormal, with frontal-parietal or frontal-temporal-parietal hypometabolism in most of the patients. A second PET scan after relief of catatonic syndrome was performed in two cases and showed significant amelioration of hypometabolism. Of the 18 case reports with SPECT, 13 were abnormal with frontal or temporal hypometabolism found in 10 patients. Amelioration after the end of the catatonic episode was seen in 9 cases (3 patients with benzodiazepine or zolpidem, 2 with antipsychotics, 1 with prednisolone during meningitis, 2 after ECT and 1 after immunotherapy in an anti-NMDAR encephalitis). All the case reports are detailed in Supplementary Tables 1 and 2. 


\section{Group studies}

Table 1 summarises all the group studies.

\section{Structural imaging}

\section{Head computerized tomography}

Four studies reported results of a CT scan of patients with a diagnosis of catatonia. Joseph and colleagues (Joseph et al., 1985) presented 5 head CT scans of patients with a diagnosis of catatonia, compared to 5 patients matched for age, gender, ethnicity, and neurologic or psychiatric diagnosis except catatonia. Three of the catatonic patients had vermis atrophy, and three had brainstem atrophy. In 1991, Wilcox (Wilcox, 1991) compared the head CT scans of 17 patients with catatonic syndrome with 30 patients with non-catatonic schizophrenia, 20 with bipolar disorder, and 15 controls. Twenty-nine percent of patients with catatonia had cerebellar atrophy, compared to $8 \%$ of schizophrenia patients, $5 \%$ of bipolar disorder patients, and $0 \%$ in healthy controls. In 1999 (Georg Northoff et al., 1999b) Northoff published a comparison of 37 brain CT scans of patients with catatonic schizophrenia, with 28 subjects with hebephrenic (or disorganised) schizophrenia and 39 with paranoid schizophrenia. He found an enlargement of cerebrospinal fluid spaces in schizophrenia patients with catatonia, especially in the left frontal-temporal regions, which was correlated with illness duration. Medda (Medda et al., 2015) in 2015 collected 26 brain imaging results from patients with catatonic syndrome and bipolar disorder; $65.4 \%$ were abnormal ( 5 enlargements of brain cerebrospinal spaces, 5 white matter lesions, 2 cerebral infarctions, 2 cortical atrophies, 1 intracranial vascular malformation, and 1 not specified abnormality), but the imaging modality (CT or MRI) was not detailed in this publication.

\section{Structural brain MRI}

There are very few published studies that reported a grouped comparison of brain MRI structural scans of patients with catatonic syndrome. Smith, in 2012 (Smith et al., 2012) reported 95 consecutive patients with catatonic syndrome in association with psychiatric disorders (affective and psychotic disorders) or general medical conditions, of which 31 had a brain MRI scan. Eighteen had diffuse cortical atrophy. It was more localized in 4 cases (bifrontal in 2 and cerebellar in 2 others). Encephalomalacia was identified in 7 cases (more frequently in patients with a history of head trauma or stroke), and multifocal lesions were seen in 3 patients (multiple sclerosis, encephalomyelitis, cerebral metastasis). In 2017, Nguien Thi Hoang published a study with 9 patients with catatonia associated with anti-NMDA receptor encephalitis (Nguyen Thi Hoang et al., 2017). Among them, 3 had a normal brain $\mathrm{CT}$, and 6 a brain MRI, of which one was abnormal showing bilateral hyperintensity in the image contrast within the medial temporal lobes. 
Recently, Hirjak et al. published an MRI study aiming to assess correlation between catatonic symptoms, cortical thickness, cortical area and local gyrification index (LGI). Data were obtained from patients with schizophrenia or schizoaffective disorder. None of these patients were treated with lorazepam or any benzodiazepines (discontinued $72 \mathrm{~h}$ before assessment). Of 87 subjects with schizophrenia or schizoaffective disorder, 25 were diagnosed with catatonia according to the NCRS (Northoff Catatonia Rating Scale) criteria. They were compared to 22 non catatonic schizophrenia patients. No difference was found in cortical thickness between schizophrenia patients with and without catatonia. Schizophrenia patients with catatonia had lower cortical area in left superior parietal gyrus, right medial orbitofrontal cortex, and lateral occipital gyrus. Patients with catatonia showed hypergyrification in the left rostral anterior cingulate and medial orbitofrontal cortex, and hypogyrification in the left superior temporal gyrus, right inferior temporal gyrus and right insula. A negative association was found between motor and behavior scores (on the NCRS) and superior frontal, insular, and precentral cortical thickness. Positive associations were found between NCRS motor and behavior scores and surface area and LGI in superior frontal, posterior cingulate, precentral and pericalcarine gyrus. According to the authors, this pattern (surface area and LGI differences between schizophrenia patients with or without catatonia) support the hypothesis of a vulnerability for catatonia among some schizophrenia patients (Hirjak et al., 2019a).

\section{Functional imaging}

\section{Magnetic Resonance Spectroscopy (MRS)}

Only one study has investigated catatonia using MRS and this was only designed to investigate levels of phosphorus metabolites. Phosphorus metabolite levels in the frontal region were found to be higher in patients with catatonic $(n=4)$, paranoid $(n=10)$ and undifferentiated schizophrenia $(n=14)$ compared to patients with disorganised schizophrenia $(n=8)$; no other differences between the groups were apparent (Shioiri et al., 1997).

\section{Single Photon Emission Computerized Tomography (SPECT)}

Four SPECT group studies were found. In a ${ }^{123}$ l lodoamphetamine (IMP) SPECT study, Satoh et al. (Satoh et al., 1993), compared 6 patients with schizophrenia who had experienced an episode of catatonia, 13 other schizophrenia patients and 7 controls and found parietal hypometabolism (particularly in posterior regions) to be associated with catatonia. All patients were asymptomatic, with chronic antipsychotic treatment, while imaging was performed. In 2000, Northoff (Northoff et al., 2000) conducted a Tc99m SPECT study including 10 catatonic patients, 10 psychiatric controls (with similar age, sex, medication and underlying psychiatric conditions but without catatonic symptoms), and 10 controls. This study was performed on asymptomatic patients one week after admission for a catatonic 
episode successfully treated with lorazepam. Northoff found a right frontal-parietal hypometabolism in patients who had experienced a catatonic episode recently. In 1999, Northoff published a ${ }^{123}$ | iomazenil SPECT study (Georg Northoff et al., 1999a) with the same 10 patients showing a decrease in GABA-A receptor density in the left sensori-motor cortex was associated with catatonia. These three SPECT studies led to the hypothesis of the implication of fronto-parietal regions and a dysregulation of the GABAergic system in catatonia. Finally, in 2000, Escobar (Escobar et al., 2000) conducted a Tc99m HMPAO SPECT study in which 9 patients (4 depressed and 5 schizophrenia patients) suffering from catatonic episode were compared before and after ECT. This study found an increased cerebral blood flow in parietal, temporal and occipital regions after successful ECT treatment in patients with mood disorders but not in schizophrenia. Patients with mood disorders needed fewer session of ECT and showed greater clinical improvement. Therefore, functional imaging during a catatonic episode could not be the same after the relief of the syndrome, which can minimize the scope of the results of the studies not performed at the time of symptom manifestations.

\section{Functional brain MRI}

\section{Motor tasks}

An fMRI study with a sequential finger opposition task conducted by Northoff in 1999 (G. Northoff et al., 1999a) on two symptomatic patients showed decreased activation of the contralateral primary motor cortex during movement. In 2009, Scheuerecker et al. (Scheuerecker et al., 2009) compared 12 schizophrenia patients with asymptomatic post-acute catatonia and found dysfunction of areas implicated in spontaneous motor actions (lesser activation in SMA and DLPFC for patients than controls).

\section{Emotional regulation task}

In 2004, Northoff and colleagues (Northoff et al., 2004) conducted an fMRI study on 10 akinetic catatonic patients compared to 10 non-catatonic patients and 10 controls. Patients were asymptomatic at the time of the study and were recruited one week after successful treatment by lorazepam of a catatonic episode. Patients and controls had to perform an emotional regulation task. This showed changes in orbitofrontal cortex (OFC) activation were associated with abnormal functional connectivity between the OFC and the medial prefrontal cortex (MPFC), and between prefrontal and motor areas. Richter (a member of Northoff's team) conducted another study in 2010 (Richter et al., 2010), on 6 asymptomatic patients who had experienced a recent catatonic episode and showed that abnormal activation of the OFC during an emotional task designed by Northoff could be treated with lorazepam. These two important studies suggested the central role of emotional regulation, functional connectivity and the GABAergic system in catatonic patients. 


\section{Resting state functional imaging}

In 2016 Walther et al. (Walther et al., 2016) compared resting-state regional cerebral blood flow (rCBF) and gray matter (GM) density in 15 patients with schizophrenia and a current episode of catatonia according to the Bush-Francis Catatonia Rating Scale (BFCRS), 27 schizophrenia patients without catatonia, and 41 healthy controls. Pseudo-continuous arterial spin labelling (ASL) sequences were used to provide an absolute measure of rCBF. Bilateral SMA and left ventral premotor cortex were the only regions in which perfusion differed between catatonic and non-catatonic schizophrenia patients, showing increased cerebral blood flow in catatonic patients. Catatonia severity was positively associated with bilateral SMA and left VPMC perfusion. Moreover, the SMA was more activated in retarded catatonia than in excited forms. The retarded form was defined by the presence of motor retardation (immobility, stupor, mutism, staring, echopraxia, echolalia, rigidity, negativism and withdrawal). The excited form was defined by the presence of motor excitation (excitement, grimacing, stereotypy and verbigeration). Finally, when compared to other schizophrenia patients, more severe catatonia was associated with higher GM density of the cerebellum and lower GM density of the frontal cortex (superior frontal gyrus and ACC) and the right insula. This important study is the only one that included patients experiencing catatonic symptoms at the time of investigation.

In 2017, the same authors published a resting-state fMRI study including 46 schizophrenia patients who were compared to 43 healthy controls (Walther, 2017). On the same day, the patients were assessed using a motor battery consisting of the BFCRS and the Modified Rogers Scale. Principal component analysis led to 4 different factors (primary motor, catatonia and dyskinesia, coordination, and spontaneous motor activity). Catatonia and dyskinesia were associated with increased thalamocortical connectivity between bilateral M1 and the left thalamus in patients with treated schizophrenia. The authors suggested that, in this case, the increased functional connectivity could be associated with motor inhibition. According to Walther et al. (Walther et al., 2019), this finding is supported by another investigation in patients with chronic periodic catatonia. Indeed, in 2018, Foucher et al. in 2018, conducted a rCBF study in 20 periodic catatonia patients, compared to 9 subjects with catatonia and 27 healthy controls. Regional cerebral blood flow was increased in the left motor and premotor areas in patients with periodic catatonia. Nevertheless, the diagnostic frame of periodic catatonia is difficult to apply to current diagnostic criteria of catatonic syndrome, and refers to the classification of Wernicke-Kleist-Leonhard. Accordingly, periodic catatonia mainly overlaps with the DSM-5 diagnosis of bipolar disorder or schizoaffective disorder (Foucher et al., 2018).

Finally, Hirjak and colleagues, in 2019, collected and analysed resting-state fMRI and structural MRI data in 87 subjects with schizophrenia or schizoaffective disorder, with or without catatonia according to NCRS, aiming to investigate the associated patterns of brain function (intrinsic neural activity) and 
structure (grey matter volume) with a multivariate analysis approach. By using a categorical approach (comparing 24 patients with schizophrenia and catatonia, and 22 schizophrenia patients without catatonia), these authors found that catatonia was associated with fronto-thalamic and cortico-striatal abnormalities. In a dimensional approach they found that behavioural symptoms were associated with cerebellar and prefrontal/cortical motor regions, and affective symptoms according to NCRS correlated with frontoparietal functional abnormalities (Hirjak et al., 2019c).

\section{Positron Emission Tomography (PET)}

Only one study has used PET specifically to investigate catatonia (Lauer et al., 2001). This study investigated three patients with the Wernicke-Kleist-Leonhard diagnostic entity of speech-sluggish catatonia, but it is unclear whether they would meet more commonly used criteria for catatonia. F-18FDG-PET in all three patients showed bilateral temporal hypometabolism compared to controls, while F-18-DOPA PET showed abnormal lateralisation in one patient. Three further studies have each included a single catatonic patient as part of a cohort of patients with schizophrenia who were undergoing F-18-DOPA PET (Dao-Castellana et al., 1997, p.; Hietala et al., 1999, 1995). Intriguingly, all three studies showed a markedly reduced DOPA binding affinity in the putamen (and in one study the caudate nucleus) when compared to other patients with schizophrenia or healthy controls. 


\section{Discussion}

We have presented the most extensive systematic review published on brain imaging in catatonia, including the analysis of 137 case reports (161 individual patients) and 18 group studies representing 186 individual patients with catatonia.

The analysis of case reports highlights that very disparate lesions can be associated with catatonia, including diffuse or focal abnormalities, which can occur in almost any brain region. Brain imaging is abnormal in the majority of cases, but abnormalities are more frequently diffuse than localised. Most of the case reports of functional imaging (PET and SPECT) are abnormal, usually showing frontal, temporal, or basal ganglia hypoperfusion. In numerous cases, a further functional neuroimaging assessment after clinical improvement showed at least partial normalisation, as also illustrated by Escobar's SPECT study (Escobar et al., 2000).

Group studies were also analysed. Two head CT studies (Joseph et al., 1985; Wilcox, 1991) showed the potential implication of the cerebellum in the pathophysiology of catatonia and the CT head study of Northoff et al. (Georg Northoff et al., 1999b) showed a more extensive diffuse cerebral atrophy in schizophrenia patients with catatonia than in other schizophrenia patients. To date, structural brain MRI studies are very scarce in the catatonia literature, mostly showing diffuse cerebral atrophy. The majority of the case reports show diffuse lesions of white matter, in a wide range of brain regions. SPECT group studies show hypoperfusion in frontal and parietal regions, correlated with the severity of catatonia. Hypoactivation in a ${ }^{123}$ I iomazenil SPECT study suggested implication of the GABAergic system (Georg Northoff et al., 1999a). An fMRI study of Scheuerecker et al. shows hypoactivation of the contralateral motor cortex during spontaneous motor task with hypoactivation of SMA in asymptomatic schizophrenia patients who have had a catatonic episode (Scheuerecker et al., 2009). Northoff's group's studies show an abnormal regulation of negative emotions with hypoactivation of OFC and hyperactivation of median PFC, associated with GABAergic dysregulation after a catatonic episode. The studies of schizophrenic patients that have included subjects with catatonia suggest that hypodopaminergia may be present in catatonia, but no studies have investigated this hypothesis as yet.

However, our study suffers from several limitations. Concerning clinical cases, there is the potential for publication biases in the literature. Case reports are usually published to discuss a clinical issue of interest. In the case of catatonia, this increases the probability of presenting a case in which brain imaging is abnormal. The few structural imaging group studies we have reported confirm the high frequency of brain abnormalities in this population. However, high quality data are lacking to 
accurately assess the proportion of abnormalities found on brain imaging for this indication. In terms of group studies, we used the keyword "imaging", which exposes us to the risk of missing articles that would only include the imaging modality in their title or abstract (head CT, fMRI...). However, a careful search within the reference sections allowed us to identify a large number of publications. Finally, another limitation, which concerns both clinical cases and group studies, is the use by the authors of different diagnostic criteria. As we pointed out in the introduction, diagnostic criteria have an influence on the estimated prevalence of the pathology. Overall, this limits the comparability of the different studies we have presented, also limiting the possibility of extrapolating mechanistic hypotheses.

Brain imaging studies have allowed authors to elaborate hypotheses of the pathophysiology of catatonia. In the seminal model developed by Northoff (Georg Northoff, 2002; G. Northoff, 2002; Northoff, 2000), catatonic symptoms are mainly associated with orbitofrontal-prefrontal/parietal cortical dysfunction and an abnormal "horizontal" cortical-cortical modulation. According to this model, catatonia is associated with GABA-ergic orbitofrontal deficits following negative emotional processing; there is abnormal connectivity between prefrontal and motor areas, resulting in a frontal lobe syndrome and motor anosognosia (i.e. that the patient is unaware of his or her own motor activity). This dysconnectivity could lead to alteration of the caudate and other basal ganglia nuclei via cortical-subcortical loop dysfunction. This hypothesis is based on the fact that, according to Northoff, the supplementary motor area (SMA) is not impaired in catatonia, unlike in "pure" motor corticalsubcortical conditions like in Parkinson's disease or in neuroleptic malignant syndrome (NMS). Moreover, Northoff et al. have demonstrated that emotional dysregulation is associated with OFC hypoactivation and he also argues that there are clinical differences between the cogwheel rigidity of Parkinson's disease and the waxy flexibility of catatonia. According to this model, the motor anosognosia of catatonia is associated with dysfunction of the right ventrolateral and dorsolateral prefrontal cortex and right posterior parietal cortex dysfunction. This model can also explain the importance of the frontal symptoms (such as stereotypies, echolalia and echopraxia) in catatonia. In this model, the reduction in the GABA-A receptor density in patients with catatonic syndrome and the efficacy of benzodiazepines suggest GABA-ergic dysfunction.

However, there are several limitations to Northoff's model. Indeed, studies have found hypoactivation of the SMA in catatonic patients with schizophrenia both in the resting state and in spontaneous movements (Scheuerecker et al., 2009; Walther et al., 2016). Interestingly, as mentioned in the introduction, clinical observations after lobotomies and frontal lobe lesions, and experiments performed on non-human primates have highlighted the specificity of supplementary motor area (SMA) dysfunction in the occurrence of waxy flexibility and negativism (Dziadziuszko et al., 2014; Joseph, 1999; Vancaester and Santens, 2007). According to Walther, hyperactivation of the SMA in 
catatonia is secondary to blockade of the cortical-subcortical loop, at a subcortical level. The procatatonic effects of antidopaminergic drugs and PET studies suggesting reduced dopamine in basal ganglia nuclei also support the importance of subcortical dysfunction in catatonic syndrome. Interestingly, these two main hypotheses about catatonia are based mainly on pharmacological and clinical arguments and the brain imaging results seem to account for a limited part of the argumentation, especially in Northoff's model. Moreover, this model has been criticised because lesions of diverse causes and localisations, including the frontal lobe, limbic system, brain stem, cerebellum and basal ganglia have also been reported to produce catatonia (Figure 2). In fact, the brain imaging studies analysed in this paper show that lesions of frontal regions and subcortical structures such as the basal ganglia can both lead to a catatonic syndrome. In the case reports collected here, some patients have only subcortical lesions, and others have only frontal lesions, with the same apparent catatonic syndrome (see supplementary materials). Indeed, we believe that catatonia is still not ready for a unified theory (Bearden and Monterosso, 2002)

A holistic hypothesis in which catatonic syndrome could be the result of two different pathways can be considered, i.e. that both frontal cortex dysregulation and "vertical" abnormal modulation of cortical-subcortical loop or cortical-subcortical primitive dysfunction can lead to catatonia. Either or both pathways can be affected. This framework allows us to give a full account of the diversity of causes of catatonic syndrome, which is considered as a final common pathway. Interestingly, Walther and colleagues recently suggest that the more recent brain imaging findings indicate an alteration in the motor system in catatonia characterized by hyperactivity within the SMA and preSMA, arguing that these structures are involved in motor control, movement selection, initiation, timing, and inhibition. According to their findings, these researchers suggest that SMA hyperactivity could result from different pathways (increased feedforward stimulation of the subthalamic nucleus, activation of other cortical areas exerting inhibitory control, or from an attempt to overcome inhibitory processes) (Walther et al., 2019).

Twenty years after Northoff's hypothesis, we are still far from proposing a fully integrated framework explaining catatonia and it seems difficult today to extract an integrative pathophysiological model of catatonia from structural and functional abnormalities of such a different nature. In fact, brain imaging studies published in the literature have serious limitations. First, most of the studies were performed on patients who had experienced catatonic syndrome but were no longer symptomatic. We may have some doubts about the appropriateness of these studies to hypothesise regarding the pathophysiology of catatonia. The abnormalities found in this kind of study could be associated with the physiopathology of catatonia, but one might also suggest that they could be sequelae of the catatonia episode, or that they could be associated with the underlying disorder more 
than a manifestation of catatonia itself; they may be trait markers of catatonia more than a state marker. Second, most of the studies have been carried out on patients suffering from schizophrenia. Yet, we still do not know if the pathophysiology of catatonic syndrome in schizophrenia is the same as that in other disorders. Third, some studies had no appropriate controls, comparing catatonic schizophrenia patients with healthy subjects (instead of non-catatonic schizophrenia patients). Fourth, most of the studies (except one of Walther's studies) were performed with patients with the akinetic form of catatonia, while excited forms have been almost entirely neglected. Due to these limitations, brain imaging studies provide very little information on the actual pathophysiology of catatonic syndrome, and the main pathophysiological hypothesis are today the same as those that were proposed before the brain imaging era (Baruk and de Jong, 1930). Given the growing literature of cases of inflammatory disorders associated with catatonia and the specific association with NMDAR encephalitis, the role of brain inflammation and glutamatergic dysfunction has been suggested as a novel line of enquiry. (Rogers et al. 2019 Lancet Psychiatry) Recommendations for future research are summarised in Box 2.

Finally, we consider that clinical and diagnostic issues remain to be resolved in order to advance the understanding of the pathophysiology of catatonia. Hirjak and colleagues (including Northoff as the last author) recently published a review of brain imaging studies in catatonia. According to these authors, brain imaging studies using criteria based on behavioural and motor symptoms mainly found implication of cortico-subcortical pathway for the pathophysiology of catatonia. Meanwhile, brain imaging studies using criteria including behavioural, motor and also affective symptoms (like the NCRS) mainly highlighted the importance of cortical regions like the orbito-frontal cortex and long-distance communication between cortical regions. These regions are largely modulated by GABAergic system, which is for the authors in line which the empirical observation of the efficacy of GABAergic treatment in catatonia (Hirjak et al., 2019b). We believe that in recent narrative reviews the hypotheses, interpretations and models of the pathophysiology of catatonia are more driven by initial conceptual frameworks than direct conclusions from brain imaging studies. This discrepancy between different brain imaging studies raises the debate of what catatonia really is. We also believe that to understand and illustrate this discrepancy, it is important to raise the question of the nature of neuroleptic malignant syndrome (NMS). In fact, for numerous authors, neuroleptic malignant syndrome appear as a manifestation of catatonia in a potentially lethal drug-induced form (Carroll, 2000; Fink, 2013; Fricchione et al., 2000). For Northoff, NMS differs from catatonia by lacking the affective dimension (G. Northoff, 2002). This important conceptual perspective has a major impact on models that we can conclude from brain imaging studies. It is also worth noting that using DSM-5 criteria, traditionally using an atheoretical approach, and without any affective symptoms for the 
definition of catatonia, NMS appears as form of catatonia (American Psychiatric Association et al., 2013).

In conclusion, catatonia is often associated with structural brain imaging abnormalities, such as diffuse white matter abnormalities and frontal lobe or basal ganglia abnormalities. During catatonic episodes, functional imaging shows hypometabolism in the frontal and temporal lobes, with hyperactivation of the SMA in catatonia associated to schizophrenia. This is consistent with work showing that psychomotor poverty in schizophrenia is associated with reduced regional cerebral blood flow in an extensive region of the pre-frontal cortex. (Liddle et al., 1992 BJPsych) These abnormalities seem to be alleviated after successful treatment of clinical symptoms. Group studies assessing brain imaging after catatonic episodes show that emotional dysregulation is related to the GABAergic system, with hypoactivation of the OFC, hyperactivation of the medial PFC, and dysconnectivity between frontal and motor areas. Asymptomatic patients with catatonia who have had catatonic symptoms may have hypoperfusion in the contralateral motor cortex during spontaneous movement. Finally, most of brain imaging studies have serious limitations. Catatonia is a recently described syndrome that still fascinates clinicians and researchers. It is a complex, severe and frequent condition. Nevertheless, the understanding its pathophysiology remains in its infancy. Brain imaging studies published so far suffer from serious limitations and for now the different models presented in the literature do not explain most of the cases. There is an important need for further studies. We propose here a new integrative model, which could guide future research endeavours. 


\section{References}

American Psychiatric Association, American Psychiatric Association, DSM-5 Task Force, 2013. Diagnostic and statistical manual of mental disorders: DSM-5. American Psychiatric Association, Arlington, $\mathrm{Va}$.

Baruk, H., de Jong, H., 1930. La catatonie expérimentale par la bulbocapnine. Masson, Paris.

Bearden, C.E., Monterosso, J.R., 2002. Catatonia isn't ready for a unified theory. Behav. Brain Sci. 25, 579-580. https://doi.org/10.1017/S0140525X02240108

Carroll, B.T., 2000. The universal field hypothesis of catatonia and neuroleptic malignant syndrome. CNS Spectr. 5, 26-33.

Dao-Castellana, M.H., Paillère-Martinot, M.L., Hantraye, P., Attar-Lévy, D., Rémy, P., Crouzel, C., Artiges, E., Féline, A., Syrota, A., Martinot, J.L., 1997. Presynaptic dopaminergic function in the striatum of schizophrenic patients. Schizophr. Res. 23, 167-174. https://doi.org/10.1016/S0920-9964(96)00102-8

Dziadziuszko, K., Szurowska, E., Pienkowska, J., Jassem, J., Dziadziuszko, R., 2014. Miliary brain metastases in a patient with ROS1-rearranged lung adenocarcinoma: a case report. J. Thorac. Oncol. 9, e34-e36.

Escobar, R., Rios, A., Montoya, I.D., Lopera, F., Ramos, D., Carvajal, C., Constain, G., Gutierrez, J.E., Vargas, S., Herrera, C.P., 2000. Clinical and cerebral blood flow changes in catatonic patients treated with ECT. J. Psychosom. Res. 49, 423-429.

Fink, M., 2013. Rediscovering catatonia: the biography of a treatable syndrome. Acta Psychiatr. Scand. 127, 1-47. https://doi.org/10.1111/acps.12038

Fink, M., Taylor, M.A., 2006. Catatonia: a clinician's guide to diagnosis and treatment. Cambridge University Press.

Foucher, J.R., Zhang, Y.F., Roser, M., Lamy, J., De Sousa, P.L., Weibel, S., Vidailhet, P., Mainberger, O., Berna, F., 2018. A double dissociation between two psychotic phenotypes: Periodic catatonia and cataphasia. Prog. Neuropsychopharmacol. Biol. Psychiatry.

Fricchione, G., Mann, S.C., Caroff, S.N., 2000. Catatonia, lethal catatonia, and neuroleptic malignant syndrome. Psychiatr. Ann. 30, 347-355.

Hietala, J., Syvälahti, E., Vilkman, H., Vuorio, K., Räkköläinen, V., Bergman, J., Haaparanta, M., Solin, O., Kuoppamäki, M., Eronen, E., Ruotsalainen, U., Salokangas, R.K., 1999. Depressive symptoms and presynaptic dopamine function in neuroleptic-naive schizophrenia. Schizophr. Res. 35, 4150.

Hietala, J., Syvälahti, E., Vuorio, K., Räkköläinen, V., Bergman, J., Haaparanta, M., Solin, O., Kuoppamäki, M., Kirvelä, O., Ruotsalainen, U., 1995. Presynaptic dopamine function in striatum of neuroleptic-naive schizophrenic patients. Lancet Lond. Engl. 346, 1130-1131.

Hirjak, D., Kubera, K.M., Northoff, G., Fritze, S., Bertolino, A.L., Topor, C.E., Schmitgen, M.M., Wolf, R.C., 2019a. Cortical Contributions to Distinct Symptom Dimensions of Catatonia. Schizophr. Bull.

Hirjak, D., Kubera, K.M., Wolf, R.C., Northoff, G., 2019b. Going Back to Kahlbaum's Psychomotor (and GABAergic) Origins: Is Catatonia More Than Just a Motor and Dopaminergic Syndrome? Schizophr. Bull.

Hirjak, D., Rashidi, M., Kubera, K.M., Northoff, G., Fritze, S., Schmitgen, M.M., Sambataro, F., Calhoun, V.D., Wolf, R.C., 2019c. Multimodal Magnetic Resonance Imaging Data Fusion Reveals Distinct Patterns of Abnormal Brain Structure and Function in Catatonia. Schizophr. Bull.

Joseph, A.B., Anderson, W.H., O'Leary, D.H., 1985. Brainstem and vermis atrophy in catatonia. Am. J. Psychiatry 142, 352-354. https://doi.org/10.1176/ajp.142.3.352

Joseph, R., 1999. Frontal lobe psychopathology: mania, depression, confabulation, catatonia, perseveration, obsessive compulsions, and schizophrenia. Psychiatry 62, 138-172.

Kahlbaum, K.L., 1874. Die Katatonie : oder das Spannungsirresein, eine klinische Form psychischer Krankheit. Berlin : Hirschwald. 
Lauer, M., Schirrmeister, H., Gerhard, A., Ellitok, E., Beckmann, H., Reske, S.N., Stöber, G., 2001. Disturbed neural circuits in a subtype of chronic catatonic schizophrenia demonstrated by F18-FDG-PET and F-18-DOPA-PET. J. Neural Transm. Vienna Austria 1996 108, 661-670. https://doi.org/10.1007/s007020170043

Medda, P., Toni, C., Luchini, F., Giorgi Mariani, M., Mauri, M., Perugi, G., 2015. Catatonia in 26 patients with bipolar disorder: clinical features and response to electroconvulsive therapy. Bipolar Disord. 17, 892-901. https://doi.org/10.1111/bdi.12348

Nguyen Thi Hoang, M., Nguyen Hoan, P., Le Van, T., McBride, A., Ho Dang Trung, N., Tran Tan, T., Nguyen Thi Thu, H., Heemskerk, D., Day, J., Vincent, A., Nguyen Van Vinh, C., Thwaites, G., 2017. First reported cases of anti-NMDA receptor encephalitis in Vietnamese adolescents and adults. J. Neurol. Sci. 373, 250-253. https://doi.org/10.1016/j.jns.2017.01.004

Northoff, G., 2002. Catatonia and neuroleptic malignant syndrome: psychopathology and pathophysiology. J. Neural Transm. 109, 1453-1467.

Northoff, Georg, 2002. What catatonia can tell us about "top-down modulation": a neuropsychiatric hypothesis. Behav. Brain Sci. 25, 555-577.

Northoff, G., 2000. Brain imaging in catatonia: current findings and a pathophysiologic model. CNS Spectr. 5, 34-46.

Northoff, G., Braus, D.F., Sartorius, A., Khoram-Sefat, D., Russ, M., Eckert, J., Herrig, M., Leschinger, A., Bogerts, B., Henn, F.A., 1999a. Reduced activation and altered laterality in two neurolepticnaive catatonic patients during a motor task in functional MRI. Psychol. Med. 29, 997-1002.

Northoff, G., Koch, A., Wenke, J., Eckert, J., Böker, H., Pflug, B., Bogerts, B., 1999b. Catatonia as a psychomotor syndrome: a rating scale and extrapyramidal motor symptoms. Mov. Disord. Off. J. Mov. Disord. Soc. 14, 404-416.

Northoff, G., Kötter, R., Baumgart, F., Danos, P., Boeker, H., Kaulisch, T., Schlagenhauf, F., Walter, H., Heinzel, A., Witzel, T., others, 2004. Orbitofrontal cortical dysfunction in akinetic catatonia: a functional magnetic resonance imaging study during negative emotional stimulation. Schizophr. Bull. 30, 405.

Northoff, Georg, Steinke, R., Czcervenka, C., Krause, R., Ulrich, S., Danos, P., Kropf, D., Otto, H., Bogerts, B., 1999a. Decreased density of GABA-A receptors in the left sensorimotor cortex in akinetic catatonia: investigation of in vivo benzodiazepine receptor binding. J. Neurol. Neurosurg. Psychiatry 67, 445-450.

Northoff, G., Steinke, R., Nagel, D., Czerwenka, C., Grosser, O., Danos, P., Genz, A., Krause, R., Böker, H., Otto, H.J., others, 2000. Right lower prefronto-parietal cortical dysfunction in akinetic catatonia: a combined study of neuropsychology and regional cerebral blood flow. Psychol. Med. 30, 583-596.

Northoff, Georg, Waters, H., Mooren, I., Schlüter, U., Diekmann, S., Falkai, P., Bogerts, B., 1999b. Cortical sulcal enlargement in catatonic schizophrenia: a planimetric CT study. Psychiatry Res. Neuroimaging 91, 45-54.

Richter, A., Grimm, S., Northoff, G., 2010. Lorazepam modulates orbitofrontal signal changes during emotional processing in catatonia. Hum. Psychopharmacol. Clin. Exp. 25, 55-62.

Sarkar, S., Sakey, S., Mathan, K., Bharadwaj, B., Kattimani, S., Rajkumar, R.P., 2016. Assessing catatonia using four different instruments: Inter-rater reliability and prevalence in inpatient clinical population. Asian J. Psychiatry 23, 27-31. https://doi.org/10.1016/j.ajp.2016.07.003

Satoh, K., Suzuki, T., Narita, M., Ishikura, S., Shibasaki, M., Kato, T., Takahashi, S., Fukuyama, H., Ohnishi, H., Morita, R., 1993. Regional cerebral blood flow in catatonic schizophrenia. Psychiatry Res. Neuroimaging 50, 203-216.

Scheuerecker, J., Ufer, S., Käpernick, M., Wiesmann, M., Brückmann, H., Kraft, E., Seifert, D., Koutsouleris, N., Möller, H.J., Meisenzahl, E.M., 2009. Cerebral network deficits in post-acute catatonic schizophrenic patients measured by fMRI. J. Psychiatr. Res. 43, 607-614.

Shioiri, T., Someya, T., Murashita, J., Kato, T., Hamakawa, H., Fujii, K., Inubushi, T., 1997. Multiple regression analysis of relationship between frontal lobe phosphorus metabolism and clinical 
symptoms in patients with schizophrenia. Psychiatry Res. Neuroimaging 76, 113-122. https://doi.org/10.1016/S0925-4927(97)00064-4

Smith, J.H., Smith, V.D., Philbrick, K.L., Kumar, N., 2012. Catatonic disorder due to a general medical or psychiatric condition. J. Neuropsychiatry Clin. Neurosci. 24, 198-207. https://doi.org/10.1176/appi.neuropsych.11060120

Solmi, M., Pigato, G.G., Roiter, B., Guaglianone, A., Martini, L., Fornaro, M., Monaco, F., Carvalho, A.F., Stubbs, B., Veronese, N., Correll, C.U., 2018. Prevalence of Catatonia and Its Moderators in Clinical Samples: Results from a Meta-analysis and Meta-regression Analysis. Schizophr. Bull. 44, 1133-1150. https://doi.org/10.1093/schbul/sbx157

Vancaester, E., Santens, P., 2007. Catatonia and neuroleptic malignant syndrome: two sides of a coin? Acta Neurol. Belg. 107, 47.

Walther, S., 2017. Aberrant resting state connectivity in the motor system indicates abnormal motor behavior in schizophrenia.

Walther, S., Schäppi, L., Federspiel, A., Bohlhalter, S., Wiest, R., Strik, W., Stegmayer, K., 2016. RestingState Hyperperfusion of the Supplementary Motor Area in Catatonia. Schizophr. Bull. sbw140.

Walther, S., Stegmayer, K., Wilson, J.E., Heckers, S., 2019. Structure and neural mechanisms of catatonia. Lancet Psychiatry.

Walther, S., Strik, W., 2016. Catatonia. CNS Spectr. 21, 341-348. https://doi.org/10.1017/S1092852916000274

Wilcox, J.A., 1991. Cerebellar atrophy and catatonia. Biol. Psychiatry 29, 733-734. 